EOMmun Communication et organisation

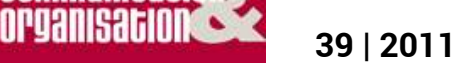

Les applications de la sémiotique à la communication des organisations

La mise en récit de la relation au client par l'entreprise Le cas de la communication d'EDF

Fabien Bonnet

(2) OpenEdition

Journals

Édition électronique

URL : http://journals.openedition.org/communicationorganisation/3169

DOI : 10.4000/communicationorganisation.3169

ISSN : 1775-3546

Éditeur

Presses universitaires de Bordeaux

Édition imprimée

Date de publication : 1 juin 2011

Pagination : 243-252

ISBN : 978-2-86781-744-1

ISSN : $1168-5549$

Référence électronique

Fabien Bonnet, «La mise en récit de la relation au client par l'entreprise Le cas de la communication d'EDF », Communication et organisation [En ligne], 39 | 2011, mis en ligne le 01 juin 2014, consulté le 19 avril 2019. URL : http://journals.openedition.org/communicationorganisation/3169 ; DOI : 10.4000/ communicationorganisation.3169 


\title{
La mise en récit de la relation au client par l'entreprise Le cas de la communication d'EDF
}

\author{
Fobien Bonnet
}

\section{La communication d'EDF : un récit d'entreprise}

L'objectif de cet article est d'envisager, du point de vue des Sciences de l'Information et de la Communication, les évolutions de la communication adressée par EDF à sa clientèle grand public. Cela implique ici une démarche de recherche spécifique cherchant à rendre compte des processus de communication observés dans leurs dimensions à la fois rationnelle, sensible et symbolique $^{2}$. Au cours de son histoire, débutée en 1946 avec la nationalisation du secteur de l'électricité et du gaz en France, EDF a produit de très nombreux messages, de natures différentes, dans des contextes eux-mêmes divers et cette hétérogénéité apparente rend délicate toute appréhension de la communication entrepreneuriale en tant que processus d'élaboration de sens supposant une forme de continuité.

Dans ces conditions, il paraît impossible de considérer le discours de l'entreprise EDF pour lui-même, indépendamment de son contexte de diffusion. En particulier, il semble nécessaire de tenir compte de ses antécédents, c'est-à-dire des messages qui ont été diffusés auparavant et des représentations que ceux-ci ont suscitées chez leur public. Face à ces exigences, nous proposons d'appréhender les différents messages émis par EDF à l'intention du grand public comme constitutifs d'une mise en scène dynamique de l'entreprise, que nous appréhenderons sous l'angle du récit.

En effet, comme le rappelle Bruno Ollivier ${ }^{3}$, le modèle de la grammaire générative " postule deux niveaux successifs dans la génération du texte, celui de la structure profonde, correspondant à des représentations abstraites qui peuvent donner lieu à différentes formes, et celui de la structure de surface,

1. Fabien Bonnet est doctorant en Sciences de l'Information et de la Communication au Celsa IUniversité Paris IV Sorbonne) sous convention Cifre avec la direction Recherche et Développement d'EDF. Son travail de recherche porte sur la mise en récit de la relation au client par les entreprises énergéticiennes et sur son adaptation au contexte sociétal.

2. Bougnoux, Daniel (1993), Sciences de l'information et de la communication, Paris, Larousse.

3. Ollivier, Bruno (2007), Les sciences de la communication, Paris, Armand Colin, p. 56. 
de ce qui est observable ». Si l'on s'en tient à cette position, l'analyse de l'enchaînement de signes, en se limitant à la structure superficielle, ne peut rendre compte du processus d'élaboration du sens sans tomber dans l'illusion d'une immanence de celui-ci. Il est donc nécessaire de se pencher sur le tissu de représentations mobilisées et suscitées par le discours, lesquelles relèveraient de la structure profonde.

$\mathrm{Si}$, comme l'écrit Sandra Jovchelovitch ${ }^{4}$, dans la mesure où elles impliquent des relations dialogiques, il est impossible de réduire les représentations sociales à une re-présentation du réel, se pose tout de même la question du rapport du texte à la réalité, rapport qu'il est notamment possible d'appréhender à travers la notion de mimesis telle qu'elle est envisagée par Paul Ricoeur ${ }^{5}$. En effet, comme le rappelle Nicole d'Almeida ${ }^{6}$, Ricoeur distingue la mimesis I, «re-présentation », de la mimesis II, « opération de configuration transformant les événements en une histoire sur le mode du « comme si » ». De même, la « mimesis III » correspondrait « au lieu de recoupement entre monde du texte et mode du lecteur/auditeur ».

En nous appuyant sur le principe de la mimesis II, il est possible d'envisager les discours produits par des entreprises comme participant d'un processus de mise en récit de leurs activités, de leurs relations avec les diverses parties prenantes et une telle approche nous semble autoriser une analyse du discours d'EDF fondée sur les représentations liées à l'activité communicationnelle de l'organisation, à son contexte et à sa dynamique. Comme le souligne encore Nicole d'Almeida dans son ouvrage consacré aux " récits économiques », «L'objet d'étude n'est pas la parole au travail ou la parole du travail, mais la parole de l'organisation, cette parole instituant un temps et un sens ». À travers une analyse du récit proposé par EDF au sujet de sa relation aux clients, nous chercherons à identifier ce temps et ce sens proposé par l'entreprise à son public.

\section{EDF et le service public. Force et pérennité d'un récit}

D'un point de vue méthodologique et afin de décrire ce récit élaboré par $\mathrm{EDF}$, nous chercherons à en déceler les inflexions, les bifurcations narratives ${ }^{7}$ liées à des événements-charnières, à des jalons de l'histoire de l'entreprise et de sa relation à la clientèle. Dans cette perspective, nous procéderons à l'analyse

4. À ce sujet, Sandra Jovchelovitch écrit : " Qu'il y ait, sans aucun doute, dans la genèse des représentations une fonction épistémique qui cherche à connaître le monde extérieur est une chose. L'analyse des représentations va cependant au-delà puisqu'elle implique des relations dialogiques d'une part (rendant compte de sa genèsel et la fonction d'expression qui travaille les êtres psychologiques dont les identités et l'existence sociale font partie intégrante des processus représentationnels d'autre part ». Jovchelovitch Sandra (2007), " La fonction symbolique et la construction des représentations : la dynamique communicationnelle Ego/Alter/Objet», in Hermes $n^{\circ} 41$, Psychologie sociale et communication, CNRS Editions.

5. Ricoeur, Paul (1983), Temps et récit, Paris, Le Seuil.

6. D'Almeida, Nicole (2001), Les promesses de la communication, Paris, PUF, p. 10

7. Le concept de bifurcation narrative est développé par Claude Brémond dans l'ouvrage suivant : Brémond, Claude (1973), La Logique du récit, Paris, Editions du Seuil. 
d'une sélection de messages représentatifs ou annonciateurs d'évolutions majeures du discours de l'entreprise au sujet de sa relation au client.

Cette première affiche (fig. 1) a été publiée par EDF en 1949, dans un contexte d'après-guerre marqué par la reconstruction des économies européennes. La composition s'articule autour de deux dessins représentant les travaux de construction de nouveaux moyens de production électrique : un barrage hydraulique et une centrale thermique.

Un texte encadre cette illustration : «Electricité de France travaille pour fournir au pays toute l'énergie électrique dont il a besoin ». On remarque qu'il est présenté de manière assez peu uniforme,

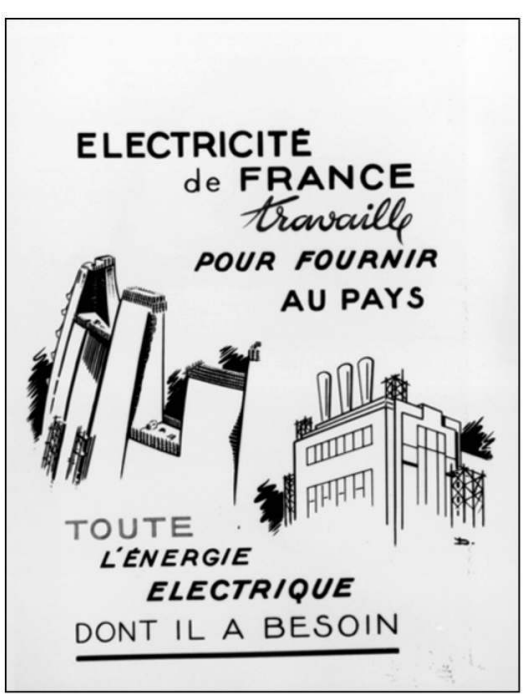

Figure 1 : Affiche EDF 1949 avec différents types et tailles de caractères. L'utilisation de différentes tailles de polices apparaît comme un moyen de hiérarchiser l'information, de donner un rythme au texte présenté en focalisant l'attention sur certains termes de l'énoncé. Les caractères sont également mobilisés en tant que métaphore visuelle, comme c'est par exemple le cas avec le mot " travaille », écrit dans une police manuscrite susceptible d'évoquer un travail manuel. Ainsi, si l'on considère la relative cohérence observée entre le choix des polices de caractère, celui de leur taille, et les différents paradigmes cités et représentés sous cette forme au sein du texte, les termes " travaille ", " énergie électrique » et "besoin » sont particulièrement mis en valeur à travers les choix typographiques. En créant une redondance entre la forme du mot et le concept évoqué, la typographie semble être utilisée pour mettre l'accent sur les notions de travail, d'énergie et de besoin.

En ce qui concerne l'illustration centrale, l'angle de vue et le type de perspective choisis mettent en valeur les éléments principaux des scènes représentées. À travers cette affiche, les travaux de construction que l'on devine entrepris par EDF apparaissent comme magnifiés, ce qui renforce leur potentiel d'évocation lié au « travail » dont il est question dans le texte. Celui-ci est représenté ici de deux façons, à travers les travaux de construction représentés, mais aussi à travers le travail de production de l'énergie nécessaire à la reconstruction, matérialisé par les centrales électriques.

C'est donc le double travail d'EDF, à la fois engagée elle-même dans la reconstruction de son appareil de production et moteur de la croissance économique de la France d'après guerre, qui est représenté sur cette affiche. Dans l'ensemble, le récit qui est proposé ici laisse transparaitre quelques traits 
du système de valeurs qui semble avoir animé la production de cette affiche. Qu'il s'agisse du discours formulé sous forme textuelle ou de celui suggéré sous forme iconographique, $\mathrm{EDF}$ se présente comme une entreprise industrielle au service du pays. L'EDF de 1949 témoigne de son investissement dans l'effort de reconstruction et affirme dans ce cadre son attachement aux valeurs de travail, de sobriété et d'engagement pour la Nation, lesquelles semblent constituer et afficher le but supra-ordonné de son action'

À travers la mise en scène d'outils de production présentés comme des monuments industriels, EDF est ici représentée comme fournissant un effort impressionnant pour rendre possible le redressement de l'économie nationale et pour inciter le citoyen à y adhérer et à y contribuer. Cette même mise en scène peut, selon nous, être appréhendée comme le point de départ d'un récit de la relation entre EDF et ses clients.

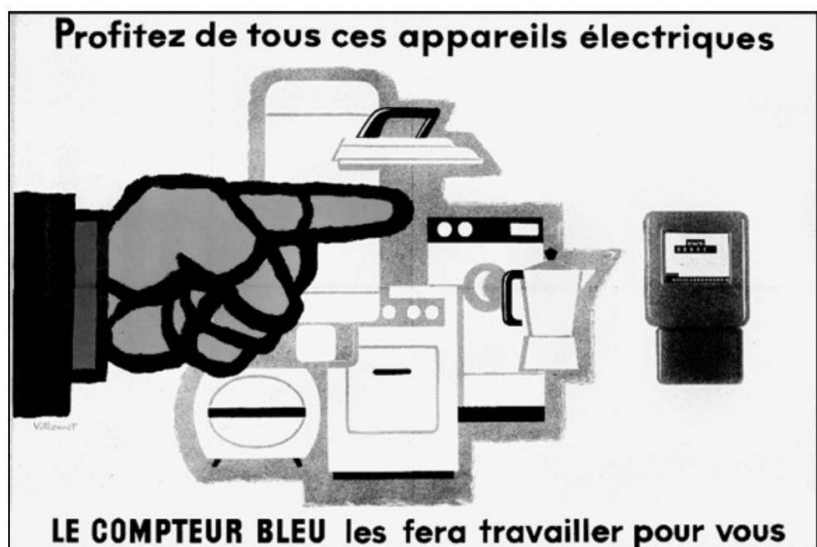

Figure 2 : Publicité EDF pour le compteur bleu (1963)

La seconde affiche dont nous souhaitons rendre compte (fig. 2) a été publiée en 1963 à l'occasion du lancement des compteurs bleus, c'est-à-dire dans le cadre d'un dispositif de grande envergure mis en place par EDF durant plusieurs années afin de procéder à une normalisation des alimentations électriques.

Selon une note interne de 1957, intitulée "Étude psychologique des attitudes du public à l'égard d'Électricité de France ", EDF jouit à cette époque d'une image très favorable auprès de ses abonnés, réputation en grande partie due aux évocations positives liées à l'électricité, teintées d'un idéal de modernité et de progrès. Cependant, une distance entre l'établissement et

8. Fischer Gustave-Nicolas (1987), Les concepts fondamentaux de la psychologie sociale, Paris, Dunod.

9. Etude psychologique des attitudes du public à l'égard d'Electricité de France (1957), note interne EDF, pp. 31-32, disponible aux archives EDF de Blois. 
son public est constatée et mise sur le compte du prestige de l'entreprise, apparemment lié à la perception de son expertise. Le caractère technique de l'électricité en fait un "objet " perçu comme complexe et hors de portée pour la majeure partie des usagers. L'abonné renonce alors à entreprendre les démarches nécessaires pour bénéficier d'une meilleure alimentation électrique et la distance symbolique qui le sépare de l'entreprise le conduit à attendre de l'aide de la part de cette dernière plutôt qu'à la mettre en cause. Finalement, l'abonné apparaît donc plutôt satisfait du service d'EDF, impressionné par les performances techniques de cette dernière, mais il se trouve dans une posture de relative "soumission " à la dimension unilatérale de la fourniture d'une offre par l'établissement public.

Dans ces conditions, en tant qu'institution respectée, reconnue pour sa compétence technique, EDF cherche à inciter ses abonnés à entreprendre les démarches nécessaires à la mise aux normes du réseau. L'objectif est de permettre à la population d'utiliser des appareils électroménagers présentés comme facteurs de "promotion sociale » dans le cadre d'une politique initiée par le gouvernement et relayée par le contrat de plan qui fixe les objectifs d'EDF. L'entreprise élabore et utilise alors un objet technique identifiable par chacun, le compteur bleu, pour matérialiser la conformité de l'alimentation aux normes exigées.

L'affiche sur laquelle nous avons concentré notre attention est signée du grand affichiste Bernard Villemot (1911-1989). Au centre de la composition apparaissent des produits électroménagers dessinés dans des teintes vertes assez diffuses. À gauche, une main, représentée à l'aide d'aplats de couleurs vives tend le doigt vers la droite et semble désigner un compteur bleu EDF, seule photographie de la composition. Ces illustrations sont encadrées par les phrases «Profitez de tous ces appareils électriques / Le compteur bleu les fera travailler pour vous".

Lillustration paraît articulée autour de trois grands ensembles signifiants : la main, l'électroménager et le compteur bleu. Cependant, la symétrie de la composition, ajoutée au choix des couleurs et à la position de la main qui passe au-dessus des appareils et désigne le compteur, semble témoigner d'une volonté d'insister sur un rapport qui reste à qualifier entre main et compteur. De plus, la phrase "Profitez de tous ces appareils électriques ", qui figure au sommet de l'affiche représente, à travers l'usage de l'impératif, à la fois une injonction et une offre, toutes deux mettant en scène l'usager dans son rapport à l'électroménager. Ce contraste entre texte et iconographie, qui ne mettent pas en avant le même type de rapport, peut être appréhendé comme une tentative de mise en scène du statut particulier du compteur. Ce dernier n'est pas l'objet initialement désiré par l'abonné mais est présenté comme un objet incontournable.

Dans l'ensemble, si l'on considère les signes présents et le contexte d'énonciation, cette affiche est porteuse d'une incitation à demander 
l'installation d'un compteur bleu dans l'optique d'une accession au confort de l'électroménager. Dans cette perspective, la dernière partie du texte confère au compteur un rôle actif : «Le compteur bleu les fera travailler pour vous ». Ce dernier est donc présenté au public sous la forme d'un dispositif engagé dans un processus de médiation entre l'usager, représenté par la main, et l'électroménager, équipement magnifié par l'énergie électrique. Cette situation de médiation au cœur de laquelle figure le compteur n'est pas sans rappeler la triangulation du désir évoquée par René Girard lorsque celui-ci écrit : « L'Homme est incapable de désirer seul. Il faut que l'objet de son désir lui soit désigné par un tiers $»^{10}$. À travers cette affiche, EDF met en scène son compteur et, par l'intermédiaire de cet objet conçu pour être porteur d'une forte charge symbolique, se met elle-même en scène en tant qu'institution offrant à l'usager une « baguette magique » source de tous les enchantements liés à la vie moderne.

Finalement, si la campagne "compteur bleu » témoigne d'une mise en récit de l'action d'EDF à travers ses compteurs, l'accent ne semble pas être mis sur une valorisation de l'action de l'entreprise. Dans les années 1960, dans le cadre d'une politique générale fixée par l'Etat, EDF cherche avant tout à développer les usages de l'électricité sans pour autant insister sur le rôle qu'elle joue dans ce développement en tant qu'institution. Respecté pour sa réputation de compétence et invité à se cantonner à ce statut, l'établissement public ne cherche pas à générer une interaction avec son public mais se lance dans une démarche qui relève d'une forme de "pédagogie " déclarative au service d'un projet d'utilité publique.

\section{Dès les années 1970, une volonté de rupture}

En 1971, à l'occasion des vingt-cinq ans d'EDF-GDF, une lettre rédigée à l'attention des abonnés, signée par la direction de l'entreprise, est jointe à la quittance adressée aux 18 millions d'abonnés que compte alors l'établissement public". Le niveau hiérarchique de ses auteurs dans l'organisation d'EDF et le fait qu'elle soit jointe à la quittance annuelle, lui confèrent un statut de document exceptionnel annonçant la portée stratégique de son contenu. En effet, ce document synthétise et concrétise les nouvelles orientations stratégiques d'EDF en ce qui concerne sa relation aux usagers. Cette réorientation sera qualifiée par la suite, de « tournant commercial $»^{12}$, ce qui témoigne de l'impact de ce message sur les politiques ultérieures.

Adressée à un très large public, cette lettre met l'accent sur une nouvelle dimension de la plus value apportée par EDF à ses abonnés, laquelle est abordée

10. Girard René (1961), Mensonge romantique et vérité romanesque, p. 13.

11. Delouvrier Paul, Hirsch Robert, Boiteux Marcel, Alby Pierre, Lettre adressée aux dix-huit millions d'abonnés d'EDF et jointe à la facture, disponible aux archives EDF de Blois.

12. Picard Jean-François, Beltran Alain, Bungener Martine (1985), Histoires de l'EDF, Paris, Dunod, pp. 223-225. 
sous l'angle d'une maximisation de l' " agrément » et non plus seulement à travers l'idée d'un accès à des appareils électroménagers porteurs de modernité et de confort. De fait, cette notion d'agrément se distingue clairement de la notion de confort dans la mesure où elle implique une évaluation personnelle et mobilise donc la subjectivité du public. En cela, elle semble se distinguer d'une vision normative du progrès.

Dans le cadre de cette redéfinition des objectifs de l'entreprise en matière de qualité de service, l'annonce d'une évolution de l' " attitude » d'EDF envers ses abonnés est particulièrement frappante. Parallèlement à la concurrence des autres énergies pour les usages domestiques et à la hausse de la productivité des centrales, ils invoquent en effet le nouveau contrat de programme signé avec l'Etat, qui « donne (à EDF) une liberté accrue, en particulier pour financer ses investissements ", pour justifier le fait qu'EDF doive adopter une " attitude commerciale plus offensive ».

Cette évolution vers une attitude " plus commerciale » était d'ailleurs déjà perceptible dès l'en-tête de la lettre à travers l'utilisation du terme "client ». En effet, le fait qu'EDF s'adresse à des clients, et non plus à des abonnés, constitue une première qui ne saurait être réduite à la seule adoption d'une nouvelle terminologie, tant elle représente une rupture symbolique dans la relation que l'entreprise publique entretient avec ses abonnés. En introduisant une notion, une figure, réservée jusqu'alors au secteur privé, EDF initie un glissement de sens qu'il est possible de considérer comme une bifurcation narrative au regard du récit qui avait été jusqu'alors progressivement élaboré par l'établissement public sans que ne soit fait appel à la subjectivité d'un client individualisé.

Vingt-sept ans plus tard, le 23 octobre 1998, un document de 31 pages intitulé "Vers le client » est présenté en Conseil d'Administration par François Roussely, nouveau président d'EDF. Son titre, complété par le sous-titre "Le compte à rebours européen ", préfigure une réflexion sur l'évolution de la relation qu'EDF entretient avec ses clients dans le cadre de la libéralisation progressive du marché européen de l'énergie. Ce document a été synthétisé à l'intention des salariés sous la forme d'une longue interview de François Roussely dans un supplément au numéro d'octobre 1998 du journal interne La vie électrique. Depuis lors, il a également fait l'objet de nombreux commentaires à l'intérieur et à l'extérieur de l'organisation's.

13. Le journal Libération avait publié un article annonçant les principaux axes du projet avant sa présentation officielle : Nathan Hervé, Schwartzbrod Alexandra, Libération, 21/10/1998, "Concurrence, nucléaire, énergies renouvelables, état-major. EDF : la réforme électrochoc ", disponible en ligne : http://www. liberation.fr/economie/0101257912-concurrence-nucleaire-energies-renouvelables-etat-major-edf-lareforme-electrochoc, dernière consultation le 16/06/2011. Le 24 octobre, Le Monde publie un autre article, signé Dominique Gallois et intitulé «EDF veut diversifier sa palette d'énergies et de services ». Plusieurs points du projet sont également détaillés dans l'étude suivante : Négocier l'emploi et la compétitivité : Etude de cas - EDF-GDF, Fondation pour l'amélioration des conditions de vie et de travail, disponible en ligne http://www.eurofound.europa.eu, dernière consultation le 15/06/2011. 
Dans le cadre de ce travail de recherche, la première partie du projet, intitulée "Tourner toute l'entreprise vers le client ", nous intéresse particulièrement. Elle commence par l'affirmation de la nécessité d'une adaptation de l'entreprise à ce qui est présenté comme une nouvelle donne en matière de relation au client, alors que s'applique progressivement la directive européenne sur le marché de l'énergie qui annonce la fin du monopole d'EDF sur le marché français. Dans cette perspective, l'accent est mis sur l'« avantage concurrentiel » dont bénéficierait EDF du fait des "valeurs de service public » qui sont les siennes. L'avenir de la relation entretenue avec les clients s'inscrirait donc d'une certaine manière dans le prolongement de la mission de service public d'EDF, c'est-à-dire dans la continuité du récit économique proposé par l'entreprise depuis des décennies.

Mais, selon les auteurs de ce projet, l'évolution du marché de l'énergie vers plus de concurrence rendrait nécessaire une compétitivité accrue d'EDF sur d'autres points, une compétitivité qui dépasserait la question d'une politique tarifaire dont on sait qu'elle a longtemps été orchestrée par le gouvernement dans le cadre d'une stratégie de développement économique du pays. Cette compétitivité étendue reposerait en particulier sur l'adoption d'une démarche accordant une importance capitale à la notion de qualité, tant en ce qui concerne les produits eux-mêmes (approvisionnement en électricité, mais aussi gaz, eau chaude, air comprimé...) que les services associés, issus d'une politique de diversification de l'offre. Il nous paraît intéressant de remarquer que la notion de qualité est ici considérée comme une nouvelle priorité stratégique d'EDF alors que l'entreprise bénéficie depuis sa création d'une image de haute technicité et de compétence, d'expertise. Comment appréhender la mise en avant de cette notion dans le discours de la direction alors que l'excellence d'EDF sur son cœur de métier est reconnue par un large public ? Est-il question de la qualité intrinsèque des produits et services proposés ou plutôt d'un souci d'adaptation aux attentes exprimées ou latentes chez le client ? Plutôt que de l'amélioration de la qualité effective, il semble qu'il s'agisse de l'intégration progressive d'une « démarche qualité » d'inspiration industrielle.

Finalement, après avoir insisté sur une évolution de la figure du client, qui ne devrait plus être considéré comme « captif» mais comme « libre et légitimement exigeant » alors que s'ouvre le marché, ce texte défend donc une prise en compte de sa subjectivité qui ne serait pas seulement cantonnée au cadre d'une mission commerciale mais devrait impliquer l'ensemble de l'entreprise. À travers ce projet, il semble que la direction d'EDF annonce une réorientation stratégique en faveur de ce qui apparaît comme une démarche marketing intégrée ${ }^{14}$, proclamant de cette façon la primauté d'une subjectivité du client.

14. «Trop de sociétés pensent qu'il incombe au seul département marketing/vente de trouver des clients et de les gérer. Pourtant, quelle que soit sa compétence, le département marketing ne peut vendre un produit mal conçu ou pallier un service déficient. II ne peut être efficace que dans un effort collectif de l'entreprise pour créer de la valeur pour les clients. ॥, Kotler Phillip (2004), Marketing Management, 11ème édition, Paris, Pearson Education, p. 68. 


\section{La mise en récit de l'entreprise : processus de signification ou panacée communicationnelle?}

La lettre aux clients de 1971 et le projet de 1998 semblent tous deux relever d'une déclaration d'intention concernant des évolutions de la relation qu'EDF entretient avec ses clients. Ces deux textes annoncent en particulier une évolution de la figure de l'abonné-client, qui deviendrait inéluctablement acteur de sa consommation d'énergie. Ils introduisent ainsi une forme de rupture avec un récit élaboré de longue date qui faisait avant tout d'EDF un acteur au service de la collectivité sans que ne soit valorisées, ou même prises en compte, l'individualité et la subjectivité de l'abonné. En faisant évoluer les figures en présence, ils suggèrent finalement un glissement de sens, d'un récit historique hérité de l'après-guerre, industriel et ouvertement politique, vers un récit plus commercial imprégné du paradigme marketing.

Appréhender les différents messages diffusés par EDF comme relevant d'un même récit nous permet de mettre en évidence la question de la continuité de la communication d'entreprise. En proclamant à plusieurs reprises l'évolution de la relation qu'elle entretient avec ses clients, en déclarant acquise la mutation de la figure même du client, EDF s'est un temps inscrite dans un modèle de communication transmissif et informationnel considérant comme acquise la volonté de chacun de s'investir dans sa consommation d'énergie. Paradoxalement, en donnant l'impression de vouloir imposer un nouveau récit potentiellement en contradiction avec les représentations élaborées au fil des ans par le public, le discours d'EDF, destiné à accompagner les clients dans le cadre d'un processus d'ouverture du marché, apparaissait comme teinté de paternalisme. Un tel constat met en évidence les risques inhérents à la pratique du storytelling, forme de déterminisme qui voudrait voir dans le récit une forme de mise en scène dont l'efficacité serait garantie, tel un outil de persuasion qui contournerait la question des représentations. Nous avons plutôt insisté ici sur l'intérêt d'une approche par les récits, intérêt qui réside selon nous dans la possibilité d'une prise en compte des processus de communication dans leur dimension dynamique, à la fois sensible et symbolique, sans faire nécessairement du récit un concept à visée strictement opérationnelle.

Résumé : Dans les années 1970, l'apparition d'une figure du client dans le discours d'EDF, en lieu et place de l'abonné invoqué jusqu'alors, témoigne d'une évolution de la manière dont l'entreprise appréhende la relation qu'elle entretient avec son public. Cet article vise à interroger cette évolution dans ses dimensions sensible et symbolique, sans négliger son caractère dynamique. Dans ces conditions, nous considérons les différents messages diffusés par l'entreprise comme participant d'un même processus de communication, que nous appréhendons sous l'angle d'une mise en récit de l'entreprise. Une telle approche nous permet de mettre en évidence l'intérêt d'une réflexion sur la 
prise en compte des représentations élaborées par le public au sujet d'une entreprise. Elle questionne également les limites d'une utilisation du récit à des fins de persuasion.

Mots-clés : Récit, Représentation, Continuité, Relation aux clients.

Abstract : In the 1970s, the emergence of the notion of customer in the discourse of EDF, replacing the former concept of subscriber, reflects a change in the way the company considers the relationship it has with its audience. This article aims to examine this evolution in both its sensible and symbolic dimensions, without omitting its dynamic characteristic. In these conditions, we consider the various announcements made by EDF as part of one same process of communication that we apprehend as a narration of the company's activity. This approach allows us to highlight the value of a reflection on the way of considering representations developed by the public about a company. It also questions the limits of using the concept of narration as a tool in order to persuade.

Keywords : Narration, Representation, Continuity, Customer relationship. 